\title{
Protecting healthcare personnel from 2019-nCoV infection risks: lessons and suggestions
}

\author{
Zhiruo Zhang ${ }^{1, *}$, Shelan Liu, ${ }^{2, *}$, Mi Xiang ${ }^{1, *}$, Shijian $\mathrm{Li}^{3}$, Dahai Zhao ${ }^{4}$, Chaolin Huang ${ }^{5}$, Saijuan Chen (凶) $)^{6}$ \\ ${ }^{1}$ School of Public Health, Shanghai Jiao Tong University School of Medicine, Shanghai 200025, China; ${ }^{2}$ Department of Infectious Diseases, \\ Zhejiang Provincial Centre for Disease Control and Prevention, Hangzhou 310000, China; ${ }^{3}$ Department of Public Health, SUNY College at \\ Old Westbury, Old Westbury, NY 11568, USA; ${ }^{4}$ School of International and Public Affairs, Shanghai Jiao Tong University, Shanghai 200025, \\ China; ${ }^{5}$ Department of Infectious Diseases, Jinyintan Hospital, Wuhan 430030, China; ${ }^{6}$ Key Laboratory of Medical Genomics, Shanghai \\ Institute of Hematology, National Research Center for Translational Medicine, Ruijin Hospital Affiliated to Shanghai Jiao Tong University \\ School of Medicine, Shanghai 200025, China
}

(C) Higher Education Press and Springer-Verlag GmbH Germany, part of Springer Nature 2020

\begin{abstract}
The outbreak of a novel coronavirus disease (COVID-19, caused by the 2019-nCoV infection) in December 2019 is one of the most severe public health emergencies since the founding of People's Republic of China in 1949. Healthcare personnel (HCP) nationwide are facing heavy workloads and high risk of infection, especially those who care for patients in Hubei Province. Sadly, as of February 20, 2020, over two thousand COVID-19 cases are confirmed among HCP from 476 hospitals nationwide, with nearly $90 \%$ of them from Hubei Province. Based on literature search and interviews with some HCP working at Wuhan, capital city of Hubei, we have summarized some of the effective measures taken to reduce infection among $\mathrm{HCP}$, and also made suggestions for improving occupational safety during an infectious disease outbreak. The experience and lessons learned should be a valuable asset for international health community to contain the ongoing COVID-19 epidemic around the world.
\end{abstract}

Keywords healthcare personnel; COVID-19; infectious diseases

\section{The outbreak}

The outbreak of novel coronavirus epidemic (COVID-19) in December 2019 resulted in a cumulative number of 80026 laboratory-confirmed cases and 2912 deaths as of March 1, 2020 [1]. Hubei Province had been hit hardest, accounting for over $83 \%$ of all the confirmed cases nationwide [1]. More than 40000 healthcare personnel (HCP) from around the country rushed there, working around the clock with local HCP in the public health intervention, diagnosis and treatment, patient care, field investigation, samples testing and sterilization [2].

Received March 4, 2020; accepted March 4, 2020

Correspondence: Saijuan Chen, sjchen@stn.sh.cn

${ }^{*}$ Zhiruo Zhang, Shelan Liu, and Mi Xiang contributed equally to this manuscript.

\section{Infection among healthcare personnel}

Sadly, as of February 20, 2020, 2055 laboratory-confirmed cases were reported among HCP from 476 hospitals across China. The majority of HCP cases $(88 \%)$ were reported from Hubei [2] (Table 1). So far, no super spreader has been identified among the HCP infections. Such a high number of infections among HCP in this ongoing epidemic is very alarming, testifying the magnitude of the epidemic, the lack of understanding of the novel virus, and the need for improvement in the medical system. Little knowledge about the novel virus led to a surge of HCP infection in the first month after the outbreak.

In addition, the sudden tide of patients in January 2020 added unprecedented stress on the municipal healthcare system of Wuhan. Healthcare staff from different medical departments in the city had to hastily take actions in a short period of time. Many did not have the required experience, knowledge, and preparedness for dealing with infectious diseases, such as proper sequence of use, replacement, and disposal of personal protective equipment (PPE). Some 
Table 1 The number of health care workers who have been infected with 2019-nCoV from December 18, 2019 to February 20, 2020

\begin{tabular}{lll}
\hline Date & $\begin{array}{c}\text { Confirmed cases from } \\
\text { Hubei, } n(\%)\end{array}$ & $\begin{array}{l}\text { Confirmed cases } \\
\text { nationwide }\end{array}$ \\
\hline December 18-31, 2019 & $0(0 \%)$ & 0 \\
January 1-10, 2020 & $19(95 \%)$ & 20 \\
January 11-20, 2020 & $281(91 \%)$ & 310 \\
January 21-31, 2020 & $906(87 \%)$ & 1036 \\
February 1-11, 2020 & $268(83 \%)$ & 322 \\
February 12-20, 2020 & $335(91 \%)$ & 367 \\
\hline Total & $1809(88 \%)$ & 2055 \\
\hline
\end{tabular}

healthcare workers had to work ten or more hours daily with no break time due to a high volume of patients and severe staff shortage. Stress and extreme fatigue could further challenge the immune system and increase the susceptibility to 2019-nCoV among HCP (Fig. 1).

The rapid expansion of the epidemic also caused a severe shortage of critical PPE. As many mass production of medical supplies was halted when workers left for family reunification at the Chinese New Year (January 25 . 2020), the healthcare system was completely caught off guard, and quickly exhausted the emergency PPE reserves. Some hospitals in Hubei had to reduce the replacement of critical PPE. The grim shortage of protective equipment greatly increased the risk of HCP infections at the onset of the outbreak, exacerbating the spread of diseases among visitors, staff, and patients.

\section{Measures taken to enhance protection of HCP}

A healthy and effective team of HCP is crucial to successfully preventing the on-going epidemic from further expansion. The high infection toll underscores the necessity and urgency of protecting HCP from COVID-19. It is laudable that the central government has attached great importance to protecting the health of HCP and has taken a number of immediate actions [3], such as improved guidance on the proper use of PPE, strengthened logistics and medical supplies, and enhanced disinfection at the hotels where HCP stay. In addition, an emergency surveillance system is now in place to monitor all exposed $\mathrm{HCP}$, contributing to prompt detection, effective triage, and isolation of infected HCP. A special medical expert group is making every effort to diagnose and treat medical staff of suspected and confirmed infection. In addition, a special health and life insurance fund is set up for all HCP working in the frontline at both national and provincial levels [4]. All of these are conducive to ensuring the confidence and efficiency of the HCP, but more needs to be done for further protection of their occupational health in the long run.

\section{Suggestions for future improvement}

The COVID-19 outbreak calls for the establishment of exposure risk assessment and management system in healthcare settings. Public health schools should be given

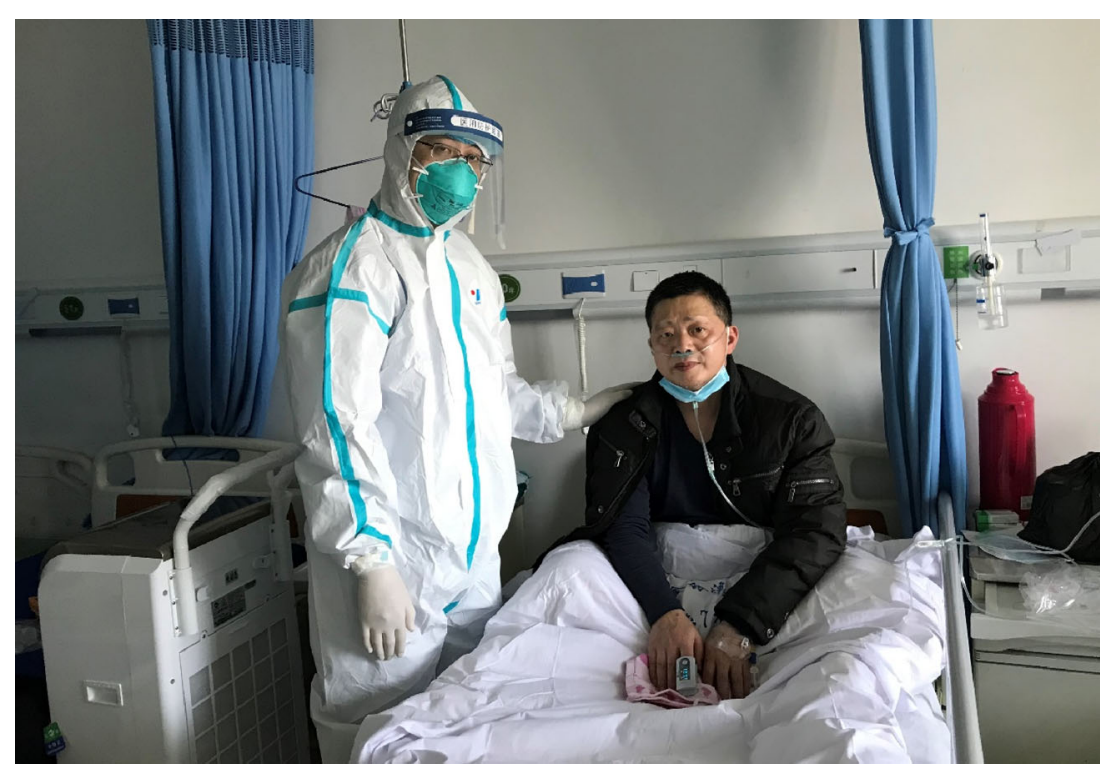

Fig. 1 Chaolin Huang, Department of Infectious Diseases, Jinyintan Hospital, Wuhan. Overworked, Dr. Huang was hospitalized for 2019-nCoV infection on January 23, and was discharged on February 17, 2020 after intensive treatment. 
more support within medical education system and all medical students should receive regular theoretical courses and practical training of public health, including the knowledge and skills to deal with large scale health emergencies, such as COVID-19. Standard guidelines and procedures should be in place to detect infectious diseases at an early stage, to timely announce the pathogens, transmission paths, diagnosis, and treatment among HCP. Furthermore, improvement in professional development as essential part of continuous medical education at all public health and medical institutions is another critical step toward reducing infection rate among healthcare workers. $\mathrm{HCP}$, regardless of the disciplines they are in, should have routine emergency drills for infectious diseases, receive professional development periodically in the protection from occupational hazards. Especially, medical staff related to the handling of infectious diseases should be well trained to properly use PPEs, and continuing education certificate can be mandatory for key HCP or staff members in all medical institutions. In addition, easy access to mental health service for HCP should be available throughout their career journey, especially during the time of crisis when they need for anxiety and stress relief.

With the stabilizing of epidemics and measures taken by the decision-makers, the shortage of PPE in China was significantly attenuated by mid-February [5]. Yet, the COVID-19 outbreak alerts us that a carefully planned stockpile of PPE and other essentials is key to effective infectious disease preparedness and to the optimal function of HCP [6]. An epidemic can affect a broad population, hence the availability and appropriate use of PPE, such as N95 respirators, face masks, gowns, and gloves, are crucial to protecting the health of HCP [7]. While it is very difficult to predict an outbreak of widespread epidemic, all the healthcare facilities should stockpile a certain amount of critical PPE to ensure an adequate supply at the onset. In addition, it is also important to set up a centralized and coordinated network of emergent PPE supply among central and local governments, healthcare facilities, and medical equipment, so as to meet the demand for consumable and durable supplies when a wide spread epidemic lasts long.

\section{Conclusions}

The ratio of confirmed HCP infection to patients is on the decrease, from $5.72 \%$ (January $11-20,2020$ ) to $2.68 \%$ (February 1-11, 2020), thanks to the increased supply of critical PPE, enhanced vigilance, and accumulated experienced among HCP [8]. It is hard to predict when and where an epidemic, like the COVID-19, will occur in the world as disease knows no national boundaries. Therefore, the compelling lessons learned in the fight against coronavirus in China must be remembered so that better public health emergency response preparedness mechanisms can be established not only in China but all over the world.

\section{Acknowledgements}

We would like to extend sincere gratitude to Mengyun Luo, Yue Fang, Kun Qian, Xueyuan Li, Jiawei Xu, and Jiahui Li who collected data.

\section{Compliance with ethics guidelines}

Zhiruo Zhang, Shelan Liu, Mi Xiang, Shijian Li, Dahai Zhao, Chaolin Huang, and Saijuan Chen declare no conflicts of interest. This manuscript is a commentary and does not involve a research protocol requiring approval by the relevant institutional review board or ethics committee.

\section{References}

1. National Health Commission of the People's Republic of China. The latest situation of novel coronavirus pneumonia. March 1, 2020. http://www.nhc.gov.cn/xcs/yqtb/202003/5819f3e13ff6413ba05fdb45b55b66ba.shtml (in Chinese) (accessed March 2, 2020)

2. WHO. Report of the WHO-China Joint Mission on Coronavirus Disease 2019 (COVID-19). 2020. https://www.who.int/docs/defaultsource/coronaviruse/who-china-joint-mission-on-covid-19-finalreport.pdf (accessed February 28, 2020)

3. National Health Commission of the People's Republic of China. Notification for further strengthening the protection of health personnel during 2019-nCoV outbreak. February 19, 2020. http:// www.nhc.gov.cn/xcs/zhengcwj/202002/75c6e88ecbeb42a9a26acb538383e2fc.shtml (in Chinese) (accessed February 19, 2020)

4. The State Council. Press Conference of the Joint Prevention and Control Mechanism of the State Council. February 20, 2020. https:// mp.weixin.qq.com/s/shefela5N9U06-6cKEIJZg (in Chinese) (accessed February 20, 2020)

5. Information Office of Hubei Provincial People's Government. Press Conference on Prevention and Control of New Coronavirus Infected Pneumonia. February 22, 2020. http://www.hubei.gov.cn/hbfb/ xwfbh/202002/t20200222_2145525.shtml (in Chinese) (accessed February 23, 2020)

6. Chang D, Xu H, Rebaza A, Sharma L, Dela Cruz CS. Protecting health-care workers from subclinical coronavirus infection. Lancet Respir Med 2020; 8(3): e13

7. Wang XH, Pan ZY, Cheng ZS. Association between 2019-nCoV transmission and N95 respirator use. medRxiv 2020; doi: 10.1101/ 2020.02.18.20021881

8. Novel Coronavirus Pneumonia Emergency Response Epidemiology Team. The epidemiological characteristics of an outbreak of 2019 novel coronavirus diseases (COVID-19) in China. Chin J Epidemiol (Zhonghua Liu Xing Bing Xue Za Zhi) 2020; 41(2): 145-151 (in Chinese) 